\title{
ISOLATION, CHARACTERIZATION AND OSTEOGENIC DIFFERENTIATION POTENTIAL OF PERIODONTAL LIGAMENT STEM CELLS (IN VITRO STUDY)
}

\author{
Marwa Sameer Moussa*, Marwa M. Abd El Hameed** and Dina Sabry****
}

\begin{abstract}
Background: Stem cells are unspecialized and immature cells that have the potential to develop into many different cell lineages via differentiation in a suitable culture media. Periodontal ligament stem cells (PDLSCs), which reside within the perivascular space periodontium, possess characteristics of mesenchymal stem cells and are a promising tool for periodontium regeneration.

Purpose: This study aimed to isolate and characterize periodontal ligament stem cells (PDLSCs) from albino rat, Identification of surface markers expression utilizing flow cytometric analysis (FACS) and assessment of the osteogenic differentiation capability of these cells.

Material and methods: PDLSCs were collected from the root surfaces of mandibular $1^{\text {st }}$ molars from 6 adult male albino rats. Collected samples were enzymatically treated and centrifuged to allow cells release. Cells were cultured for fourteen days. After reaching confluence, the isolated cells were characterized by flow cytometry using CD73, CD133 and C-Kit. The cells were induced for osteogenic differentiation by placing them in osteogenic culture media. Osteogenic differentiation was evaluated by Alizarin Red stain and by Molecular assessment for Bone sialoprotein (BSP) and alkaline phosphatase (ALP) genes expression by real time PCR (q RT-PCR).
\end{abstract}

Results: The results showed successful isolation of stem cells from periodontal ligament. PLSCs were identified as mesenchymal stem cells by expression of mesenchymal stem cell markers through FACS. PDLSCs showed positive expression to CD73 marker and negative expression to hematopoietic markers CD133 and C-kit. Our results showed successful differentiation of PDLSCs into osteogenic lineage. The formed mineralized matrix stained by alizarin red and was more advanced at 21 days after osteoblastic differentiation. Real Time PCR (q RT-PCR) for quantitative expression of BSP and ALP showed significant increase in the expression of BSP and ALP from day 14 to day 21 in differentiated PDLSCs.

Conclusion: According to the obtained results, we conclude that PDLSCs could be successively isolated from periodontal ligament tissues. Isolated PDLSCs possess an osteogenic potential and show differentiation into osteogenic lineage when cultured in suitable osteogenic medium.

KEY WORDS: Periodontal ligament stem cells (PLSCs), CD133, C-Kit, CD73, Osteogenic differentiation, BSP, ALP.

* Lecturer of Oral biology, Faculty of Oral \& Dental Medicine, Cairo University

** Lecturer of Oral biology, Faculty of Dentistry, Ain Shams University

*** Professor of Medical Biochemistry and Molecular Biology, Department of Medical biochemistry and Molecular

Biology, Faculty of medicine, Cairo University. 


\section{INTRODUCTION}

Tissue engineering gives a new era for therapeutic medicine; it is progressing in a very rapid way and extending to involve all body tissues. Three decades ago, tissue engineering was an idea and nowadays it has become a potential therapy for several conditions. ${ }^{[1]}$

Stem cells are unspecialized and immature cells that have the potential to develop into many different cell lineages via differentiation. By the conventional definition, these cells can renew themselves indefinitely through self-renewal. ${ }^{[2]}$

An important feature of tissue stem cells is that their capacity to generate other cell types is usually limited. For example, a brain stem cell can become any cell type of the brain, but not a muscle cell. Hence, they are termed multipotent; capable of producing some cell types but not all cell types in the body (in contrast to pluripotent stem cells). Embryonic pleuripotent stem cells can give rise to every other cell type in the body. ${ }^{[3]}$

Mesenchymal stem cells (MSCs) must be adherent to tissue-culture-treated plastic when they are maintained in standard culture conditions. MSCs must express CD73, CD105 and CD90 and lack the expression of CD45, CD14, CD34or CD11b, CD79a or CD19 and HLA-DR surface molecules. Additionally, MSCs must be able to undergo osteoblastic, chondroblastic and adipocytic differentiation in vitro. This is according to the criteria of International Society for Cellular Therapy (ISCT). ${ }^{[4]}$

Recent studies have revealed that the oral tissues are a rich source of stem cells. Tissue engineering is also considered to be a new field in the regeneration of missing oral tissues. ${ }^{[5]}$

Several sources of dental stem cells (DSCs) have been identified in different dental tissues, including the periodontal ligament (PDLSCs), the bone marrow from orofacial bone (BMSCs), the periosteum (PSCs), the dental pulp of permanent teeth (DPSCs), the naturally exfoliated deciduous teeth (SHED), the apical papilla (SCAP), the dental follicle (DFSCs), the tooth germ (TGPCs), the salivary gland (SGSCs), the oral epithelium (OESCs) and the gingival stem cells (GMSCs). ${ }^{[6]}$

Periodontal ligament stem cells (PDLSCs), which reside within the perivascular space of periodontium, possess characteristics of mesenchymal stem cells and they are a promising tool for periodontal regeneration. Recently, great progress has been created in PDLSCs transplantation. PDLSCs obtained from mature periodontal ligaments have stem cell properties similar to MSCs rather than neural crest cells. ${ }^{[7]}$ PDLSCs express MSC surface markers CD105, CD90 ${ }^{[6]}$ and CD73 ${ }^{[8]}$ They should also showed negative expression of hematopoietic stem cell (HSC) markers as CD45, CD34, and CD14 or CD11b, CD79a, or CD19, C-kit and HLA class II. ${ }^{[9]}$

C-kit is a membrane tyrosine-kinase receptor, which specifically interacts with the stem cell factor (SCF), is expressed by different cell types. It is expressed in hematopoietic stem cells, melanocytes, bone marrow stem cells and adipose stem cells. Moreover, it was demonstrated that c-kit expression was found in human dental pulp stem cells. ${ }^{[10]}$

CD133 is one of key biomarkers for isolation and characterization of stem cells. CD73 is not only a biomarker for isolation and characterization of stem cells, but there is an increasing evidence of its role in cell development and growth. ${ }^{[1]}$

Bone sialoprotein (BSP) is one of the major calcium binding, non-collagenous glycosylated phosphoproteins in the extracellular matrix of mineralized tissues such as bone and dentine. BSP belongs to the small family of integrin binding ligand N-linked glycoproteins. It is expressed by several cell types associated with mineralized tissues but is produced in abundance by osteoblasts. The molecule of BSP is linear with little secondary structure. However, the protein is highly flexible, containing spatially segmented motifs that can bind 
several ECM constituents with diverse biological roles, including collagen, matrix metalloproteinases, hydroxyapatite, as well as integrin present in numerous cell types. In vitro experiments with bone derived osteoblasts and bone marrow cells have revealed that BSP not only stimulates calcification of newly synthesized organic matrix but also promotes cell proliferation and expression of osteoblastic phenotypes, suggesting that BSP may play a role in osteoblast differentiation and the onset of mineral formation. ${ }^{[12,13]}$

Alkaline phosphatase (ALP), a glycoprotein belonging to a family of proteins anchored to the plasma membrane via glycosylphosphatidylinositol linkage, catalyzes the hydrolysis of phosphate esters in alkaline $\mathrm{pH} .{ }^{[14,15,16]}$

ALP is a common biochemical marker used to assess osteoblast differentiation and is considered to be involved in skeletal mineralization. ${ }^{[17,18]}$

ALP is abundant in matrix vesicles which play a role in extracellular matrix processing and calcification of bone. The precise role of ALP in mineralization remains unclear. It may be related to its calcium-binding action, generation of free phosphate, or degradation of mineralization inhibitors. ${ }^{[19]}$ Increased levels of alkaline phosphatase activity have also been reported as one of the phenotypic changes of fibroblasts in wounds and inflammation. ${ }^{[16]}$

\section{MATERIAL AND METHODS}

\section{I) Sample collection}

This work was performed in the Unit of Biochemistry and Molecular Biology at The Medical Biochemistry Department, Faculty of Medicine, Cairo University. All steps of the experiment were approved by the Ethical Committee of Experimentation on Animals of Faculty of Medicine, Cairo University. In this in vitro study, periodontal ligament samples were collected from root surfaces of 12 extracted lower $1^{\text {st }}$ molar teeth from both sides of six healthy adult male albino rats weighing from (180-220 gm).

All procedures for tooth extraction were done under general anesthesia. Once sedation and anesthesia signals were verified, humidified gauze was placed to cover the animal's eyes to avoid dryness of the corneas. The animal was then positioned on a flat board. Two rubber bands were used to maintain the mouth opened and to make the tongue immovable. Then by a scalpel, disconnecting the surrounding gingiva of the mandibular $1^{\text {st }}$ molar and the extraction was carried out. ${ }^{[20]}$

Immediately after the extraction the teeth were disinfected using disinfectant solution, to decrease the possible amount of bacterial presence. Then, the teeth fully immersed in the Hanks balanced salt solution enhanced by antibiotics and antifungals (composed of $1 \mathrm{ml}$ of Hanks balanced salt solution, $9 \mathrm{ml}$ water for inj; $200 \mu \mathrm{l} / 10 \mathrm{ml}$ gentamycin,200 $\mu \mathrm{l} / 10 \mathrm{ml}$ streptomycin, $200 \mu \mathrm{l} / 10 \mathrm{ml}$ and $200 \mu \mathrm{l} / 10$ $\mathrm{ml}$ penicillin) to fully eliminate any remaining bacterial presence. The PDL tissues were scraped from the root surfaces using a dental spatula as soon as possible (no later than $24 \mathrm{hrs}$ after extraction). ${ }^{[21]}$

\section{II) PLSCs culture and characterization:}

In sterile conditions under laminar flow hood, the excised tissues were placed in a small plate and minced into small pieces. The minced pieces were collected in sterile, labeled Eppendorf tubes to which a digesting solution of collagenase type II and dispase were added. The reaction was stopped by addition of the culture medium. The media contained the following ingredients: DMEM+100 $\mathrm{mg} / \mathrm{mL}$ penicillin, $100 \mathrm{mg} / \mathrm{mL}$ streptomycin, $10 \%$ fetal bovine serum (FBS), $5 \mathrm{mg} / \mathrm{mL}$ amphotericin B and $0.1 \%$ L-glutamine. The tubes were centrifuged to obtain a cell pellet of isolated cells. The pellet was resuspended in culture medium by successive pipetting. $1 \mathrm{ml}$ of the culture media tube was taken and placed on a hemocytometer. ${ }^{[22]}$ 


\section{Sub culturing (Passaging):}

Passaging was preformed when the primary cell culture of adherent cells reached $80 \%$ confluence and was named passage zero (P0). Following confluence, the cells were passed successfully up to the third passage (P3). Cells were sub cultured every other week and the culture medium was replaced every 2 - 3 days in a Laminar Flow safety cabinet. ${ }^{[23]}$

\section{III) Flow cytometric analysis (FACS):}

Cells were identified as being mesenchymal stem cells (MSCs) by their morphology and plastic adherence. In addition to quantification of several expressed MSCs markers via using flow cytometric analysis (FACS). Adherent cells were trypsinized and subjected to centrifugation to form cell pellet. Then $1 \times 10^{5}$ cells were incubated with $10 \mu$ of monoclonal CD73 PE, CD133PE and C-kit PE antibodies, at $4{ }^{\circ} \mathrm{C}$ in the dark. Same species isotypes served as a negative control. After 20 minutes' incubation, $2 \mathrm{ml}$ of PBS containing 2\% FCS solution were added to a tube of monoclonal treated cells. The mixtures were then centrifuged for 5 minutes at $2500 \mathrm{rpm}$ followed by discarding the

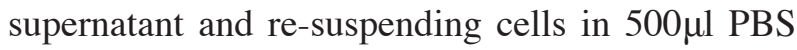
containing 2\% FCS. Cell analysis was performed using CYTOMICS FC 500 Flow Cytometer and analyzed using CXP Software version 2.2. ${ }^{[24]}$

\section{IV) MTT Assay:}

MTT Reagent supplied ready for use were obtained from (TACSTM TREVIGEN ${ }^{\circledR} \quad 8405$ Hegerman Ct. Gaithersburg). The cells were seeded in three 96-well tissue culture plates containing $10^{3}$ cells/ ml per well. The MTT reagent was added and the plate was incubated in the dark for 2 to 4 hours. The absorbance in each well was measured at a range from 490 to $630 \mathrm{~nm}$ using an enzyme-linked immunosorbent assay plate reader (Stat Fax 2200, Awareness Technologies, Florida, USA). ${ }^{[25]}$

\section{V) Osteogenic Differentiation:}

Osteogenic differentiation was induced by incubating the confluent monolayers with osteogenic induction medium of DMEM, 10\% FBS, dexamethasone (10nM), $\beta$-glycerophosphate $(10 \mathrm{mM})$ and ascorbic acid 2-phosphate $(50 \mathrm{mg} / \mathrm{mL})$ to differentiate into osteogenic lineage. The medium was replaced every two to three day. ${ }^{24]}$

After applying the osteogenic induction medium, the cells were observed regularly for morphological changes.

\section{Assessment of osteoblasts Differentiation}

\section{A) Alizarin Red Staining:}

The cultures were stained using Alizarin Red Stain. During the staining process, the samples were washed with phosphate buffer saline (PBS) and fixed in $4 \%$ buffered paraformaldehyde and were then washed with distilled water. After 10 minutes at room temperature, they were stained and washed again with distilled water and PBS at a pH of 7.2. ${ }^{[26]}$ The mineralized nodules were monitored using an inverted light microscope and digital micrographs were taken at day 14 and 21.

\section{B) Gene expression profile (Quantitative $R T-P C R)$ :}

Total RNA was isolated using RNA purification kit (Gene JET, Kit, \#K0731, Thermo Fisher Scientific Inc. Germany). RNA quantification using real-time reverse transcription polymerase chain reaction (RT-qPCR) was achieved by one step reaction (SensiFAST ${ }^{\mathrm{TM}}$ SYBR ${ }^{\circledR}$ Hi-ROX OneStep Kit, catalog no. PI-50217 V, Bioline, UK). Sequence-specific primers (Bio Basic, USA) for the studied target genes (BSP\&ALP) were used. The prepared reaction mix samples were applied in real time PCR (StepOne Applied Biosystem, Foster city, USA). We used $1 \mu \mathrm{M}$ of both primers specific for each target gene. Primers sequence and annealing temperature specific for each gene demonstrated in (table 1). RT-PCR was performed at day 14 and 21 after differentiation in accordance with Haase et al. ${ }^{[27]}$ 
TABLE (1) Primers sequence specific for each studied gene:

\begin{tabular}{cll}
\hline $\begin{array}{c}\text { Target } \\
\text { gene }\end{array}$ & \multicolumn{1}{c}{ Primer sequence: 5'-3' } & \multicolumn{1}{c}{$\begin{array}{c}\text { Gene bank } \\
\text { accession } \\
\text { number }\end{array}$} \\
\hline ALP & $\begin{array}{l}\text { F: GACCCTGAAAAATGCCCTGA } \\
\text { R: ACTTGTCCATCTCCAGCTTG }\end{array}$ & NM013059.1 \\
& $\begin{array}{l}\text { F: GGACTCCCCATTCTGTCTTTGC } \\
\text { BSPP }\end{array}$ & NM012587.2 \\
& R: AACCTCGAAGCATTCCACCTGC & \\
\hline
\end{tabular}

\section{VI) Statistical analysis}

Data were coded and entered using the statistical package SPSS (Statistical Package for the Social Sciences) version 23. Data were summarized by mean and standard deviation. P-values less than 0.05 were considered as statistically significant.

\section{RESULTS}

\section{I- Isolation:}

Stem cells were successfully isolated from periodontal ligament of lower first molar teeth of albino rats. Cells were observed on regular basis using an inverted light microscope.

\section{Periodontal ligament Derived Stem Cells:}

At the beginning of culture, after enzymatic digestion and initial seeding was confirmed, one week later, the cultured cells were attached to the plastic flask floor and exhibited fibroblast-like morphology. The cells appeared spindle shaped with extended processes (figure1a). After 2 weeks, cells showed an increase in their density. Cells still appeared as spindle shape but with extended long interconnected processes and continued to proliferate and propagate reaching $80-90 \%$ confluence by day 14 . (figure. 1b)

\section{III- Flow cytometric analysis after isolation:}

Flow cytometric analysis revealed negative expression of CD133 ( $0 \%$ )(Figure 2a), negative expression of $\mathbf{C}$-kit $(0.2 \%)$ (Figure $2 \mathrm{~b}$ ) and positive expression of CD73 (55\%) (Figure 2c).

\section{IV) MTT Assay:}

According to the statistical results, the mean value of PDLSCs count after one week is 0.69 \pm 0.282 . The mean value of PDLSCs after two weeks is $1.138 \pm 0.727$. Tissue culture plates containing $10^{3}$ cells/ ml per well.
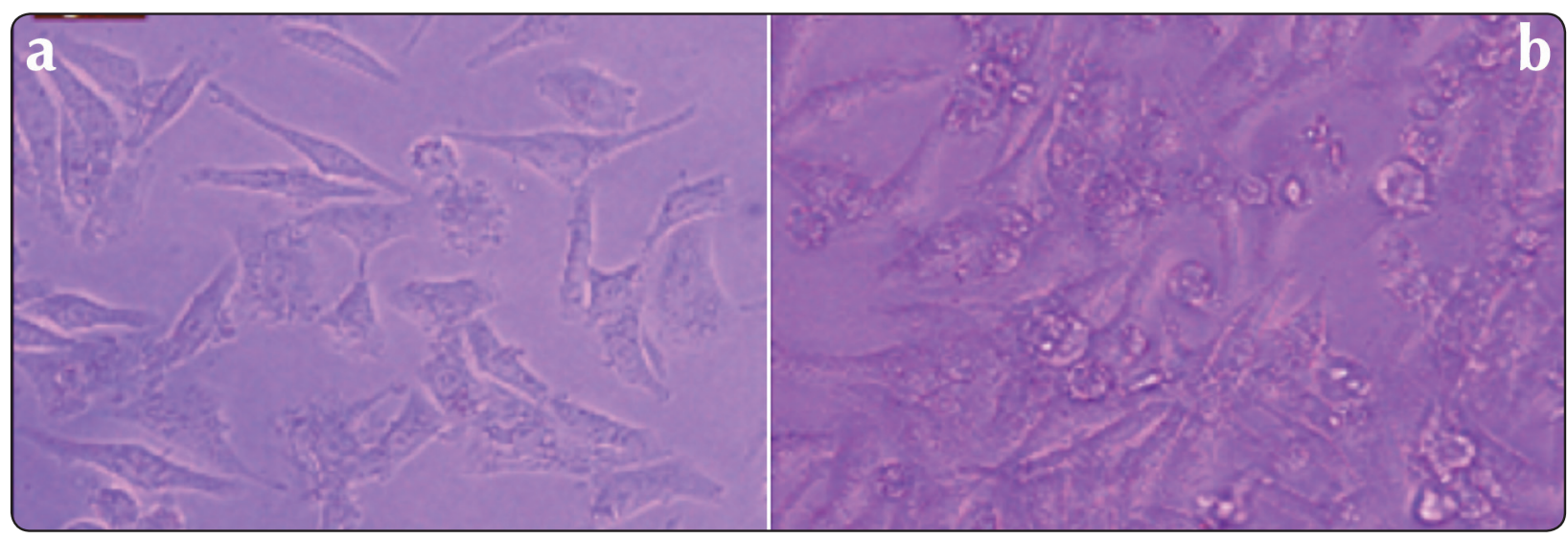

Fig. (1a) Photomicrograph of PDLSCs after one week of isolation showing spindle shape cells with extended processes (Original magnification x 200)

Fig. (1b) Photomicrograph of PDLSCs after two weeks of isolation showing increase the density of cells. Cells appear spindle shape with extended long interconnected cell processes.(Original magnification x 200) 


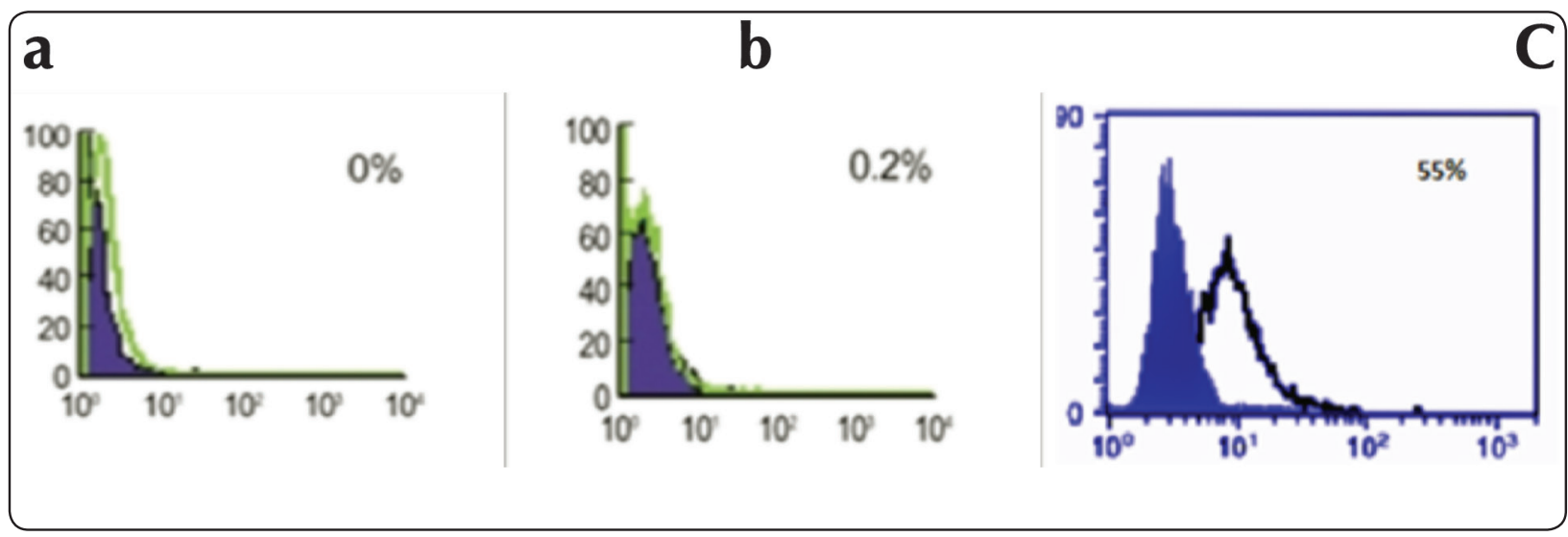

Fig. (2a) Flow cytometric analysis showing CD133 with 0\% expression in MSCs isolated from peridontal ligament. Fig. (2b) Flow cytometric analysis showing C-Kit with $0.2 \%$ expression in MSCs isolated from periodontal ligament. Fig. (2c) Flow cytometric analysis showing CD73 with 55\% expression in MSCs isolated from periodontal ligament.

MTT assay for PDLSCs after one week and two weeks showing significant increase in the proliferation of PDLSCs from week one to week two (p-value < 0.05). (Fig.3)

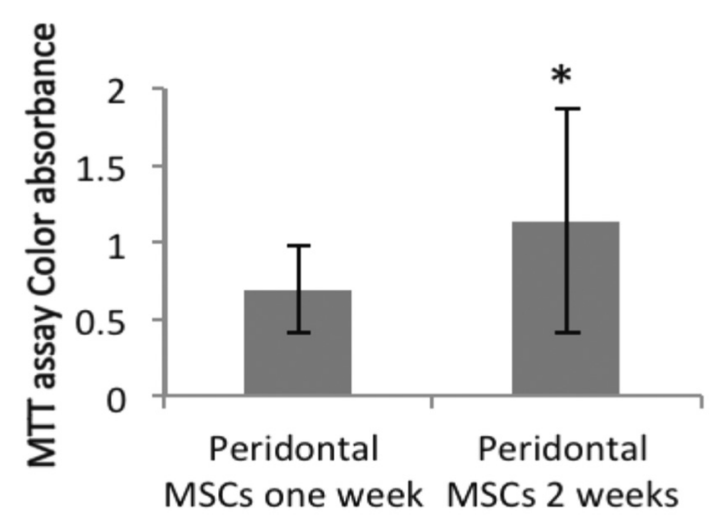

Fig. (3) Bar chart of MTT assay for PDLSCs after one week and two weeks showing significant increase in the proliferation of PDLSCs from week one to week two. *means significant difference ( $\mathrm{p}$-value $<0.05$ ).

\section{IV- Assessment of Differentiation}

\section{1-Alizarin Red Staining}

On the $14^{\text {th }}$ and $21^{\text {st }}$ days from osteogenic differentiation, cultured cells were stained with
Alizarin Red to identify nodules of calcification. After applying the osteogenic induction medium, the cells were observed regularly for morphological changes.

Within the first week after induction of osteogenic differentiation, cells within dishes started to change their spindle-shape into round shaped cells which aggregated to form clusters. Cell migration occurred mainly from the periphery towards the center of the dish, giving rise to rounded aggregates (Fig. 4a).

By the $14^{\text {th }}$ day, staining with calcium specific Alizarin Red stain was done and the aggregated cultured cells gave positive results indicating the formation of calcific deposits. (Fig. 4b). By the $21^{\text {st }}$ day, the staining became more intense and multiple isolated mineralized extracellular nodules appeared (Fig. 4c).

\section{2-RT-PCR:}

Real Time PCR (q RT-PCR) for quantitative expression of BSP and ALP showed significant increase in the expression of BSP and ALP from day 14 to day 21 in differentiated PDLSCs. (Fig. $5 \mathrm{~A} \& 5 \mathrm{~B})$. 


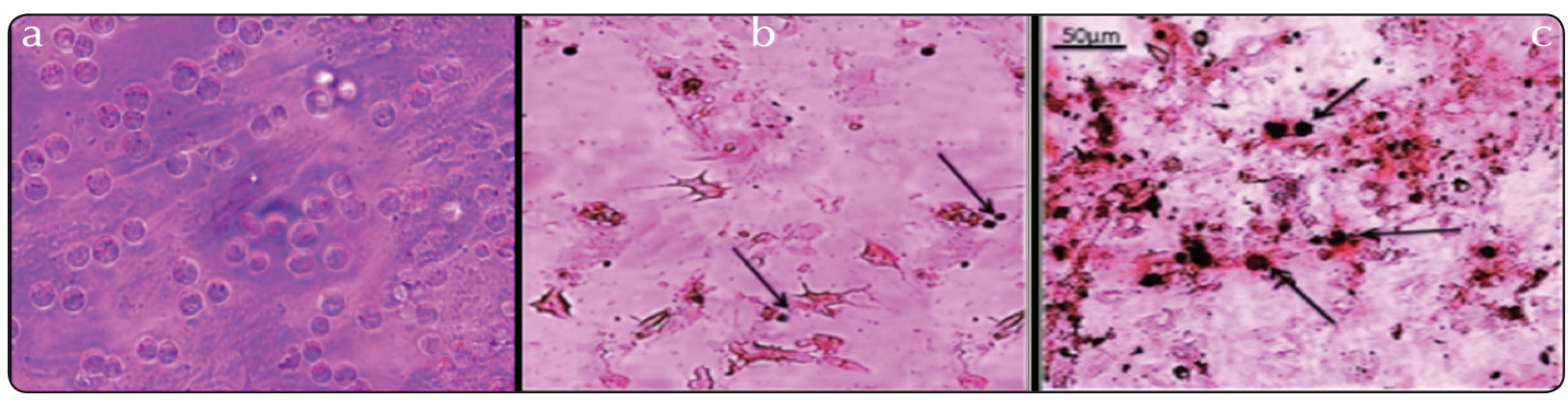

Fig. (4a) Photomicrograph of unstained PDLSCs (day 7) during osteogenic differentiation where cells lost their spindle- shape and became rounded (Original magnification x 400).

Fig. (4b) Photomicrograph of PDLSCs (day 14) stained with Alizarin Red showing extracellular calcific deposits (black arrows) (Original magnification $\mathrm{x} 400$ ).

Fig. (4c) Photomicrograph of PLSCs (day 21) stained with Alizarin Red showing multiple isolated mineralized extracellular nodules (black arrows) (Original magnification x 400).

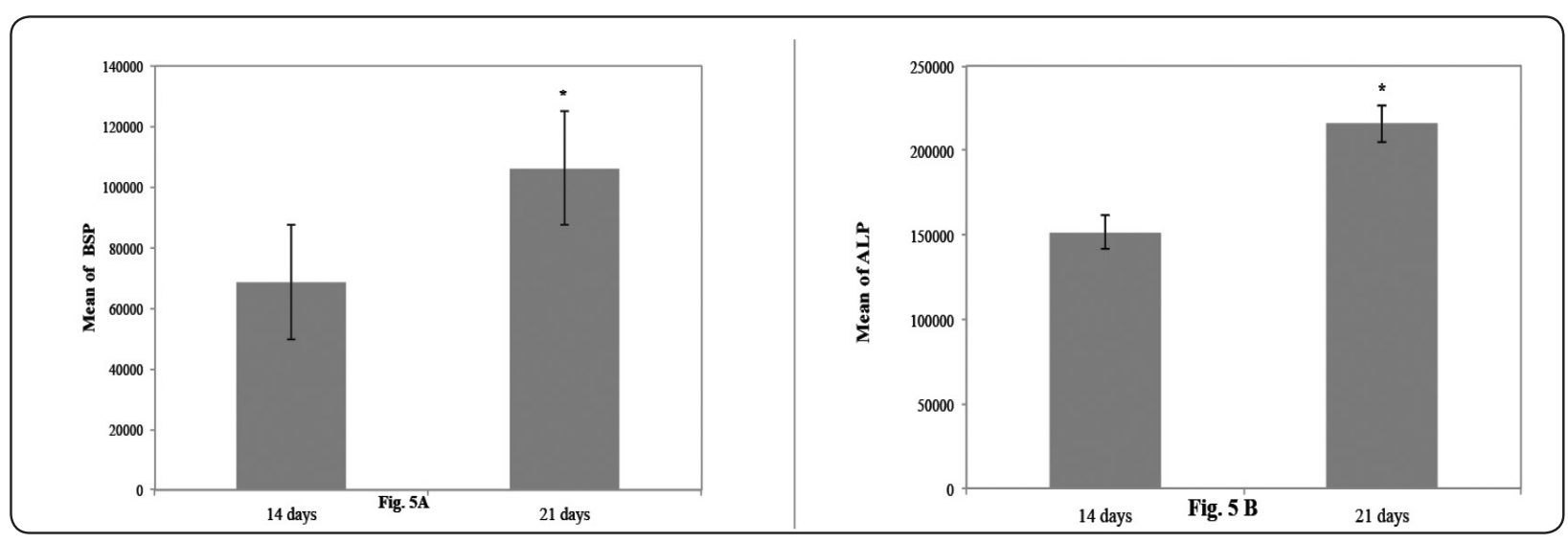

Fig. (5a) Bar chart showing significant increase in the expression of BSP from day 14 to day 21. *means significant difference (p-value <0.05).

Fig. (5b) Bar chart showing significant increase in the expression of ALP from day 14 to day 21. *means significant difference (p-value <0.05).

\section{DISCUSSION}

Regenerative medicine is a field that combines numerous aspects of medicine, materials science, cell and molecular biology and bioengineering in order to regenerate, repair or replace tissues. ${ }^{[28]}$ In the present study, the isolation of stem cells from periodontal ligament was aimed together with comparing these cells regarding their characterization and capability to differentiate into osteogenic lineage.
For the purpose of this study, PDL samples were obtained by scrapping the roots surface, a process which was previously used by many researchers. ${ }^{[29,30,31]}$ Periodontal ligament tissue inflammatory involvement is frequently observed in everyday clinical situations. Therefore, they provide more accessible source of stem cells than pulp tissue from extracted healthy teeth.

In our work, isolated periodontal ligament stem cells were able to attach to the plastic flask floor and exhibited fibroblast-like morphology after in 
vitro expansion. This was in accordance with Alge et al., ${ }^{[32]}$ as they revealed same criteria for isolated BMSCs and DPSCs from adult Sprague-Dawley rat. PLSCs were identified as mesenchymal stem cells by quantification of several expressed mesenchymal stem cell markers via using flow cytometric analysis. PLSCs were shown to be positive for CD73 marker and negative for hematopoietic markers CD133 and C-kit. These results came in accordance with Eleuterio et al. ${ }^{[33]}$ who stated that: "Cellular characterizations of the primary cultures from human dental pulp and periodontal ligament are similar to BMSCs for their high clonogenic potential and for expression of embryonic and mesenchymal markers".

Regarding the in vitro MTT proliferation results for the cultured PLSCs, maximum proliferation was achieved at 14 days. This was supported by our statistical results, which showed a significant increase in the proliferation of PDLSCs from day 7 to day 14 (p-value was <0.05). This was in agreement with Park et al. ${ }^{[24]}$ who assessed the proliferation potential of the cultured human PDLCS from day 1 to day 12 , and noticed an increase in the cell count as the time progressed.

In the current study, samples were cultured in DMEM, which has higher calcium and magnesium ions than other media such as Roswell Park Memorial Institute medium and is particularly suitable for cell growth. This medium contained 100 units/mL of penicillin to prevent the growth of gram-positive bacteria, $100 \mu \mathrm{g} / \mathrm{L}$ of streptomycin to inhibit the growth of Gram-negative bacteria, $50 \mu \mathrm{g} / \mathrm{mL}$ gentamycin to prevent the growth of Gram-negative and Gram-positive bacteria and amphotericin B antifungal drug. [29,30]

In the present study, samples were placed in osteogenic media in accordance with standard differentiation protocol. Osteogenic differentiation potential of PLSCs, making them useful in tissue engineering under suitable conditions.
In our research, we used osteogenic differentiation media contain ascorbic acid, dexamethasone and $\beta$ - glycerophosphate $(\beta-\mathrm{GP})$ to support the osteogenic differentiation of PLSCs in vitro. The mechanism of mineralization produced by $\beta$-glycerophosphate remains unclear, but it is linked to the mechanism of action of alkaline phosphatase. Alkaline phosphatase can hydrolyze organic phosphate and release inorganic phosphate which in turn can promote mineral deposition on the surface of tissue culture. ${ }^{[34]}$

Osteogenic differentiation of PDLSCs in the present study was confirmed by Alizarin red stain and by RT-PCR tests for ALP and BSP genes expression.

In the present study, Mineralized matrix formation stained by alizarin red was more advanced at 21 days after osteoblastic differentiation than at 14 days which coincides with the results of RT -PCR of gene expression of ALP and BSP of our work.

The mRNA expression of ALP was selected in the present study as ALP stimulates matrix formation and mineralization. There is a correlation between the high levels of ALP and an increase in mineralized globules through Alizarin red stain ${ }^{[35]}$. The present study showed significant increase of the expression of ALP from day 14 to day 21. This was coincident with Ohazama et al., ${ }^{[36]}$ who correlated this increase in ALP expression to the increase in extracellular matrix mineralization nodules at later stages of differentiation.

BSP is specifically expressed by fully-differentiated osteoblasts. ${ }^{[37]}$ In our research, in vitro mRNA expression of BSP suggested that PLSCs have the potential for hard tissue formation and the ability to facilitate periodontal regeneration. ${ }^{\text {[38] }}$

BSP plays an important role in osteoblast differentiation and bone mineralization. ${ }^{[38]}$

In the current study, the mRNA expression of BSP provides further evidence of the hard tissue formation potential within a stem cells residing within the periodontal ligament. 


\section{CONCLUSION}

1. According to the obtained results from the current investigation, it could be concluded that PDLSCs can be successively isolated from periodontal ligament tissues scraped from roots of lower $1^{\text {st }}$ molar teeth of albino rats. Isolated PDLSCs showed positive expression of CD73 and negative expression of CD133 and C-kit. In addition, PDLSCs possess an osteogenic potential and they produce mineralized nodules, when cultured in the proper osteogenic medium. The PCR data sheet for the mRNA showed increased positive expression of mRNA genes BSP and ALP.

2. PDLSCs may present a suitable, accessible and potential alternative source for regenerative medicine and therapeutic applications due to their differentiation potentials. However, further researches are suggested to be done on PDLSC gene expression to enhance identification of these cells and asses the possibility of getting them from human PDL sources.

\section{REFERENCES}

1. Chen, F.M. et al. A review on endogenous regenerative technology in periodontal regenerative medicine. Biomaterials. 2010; 31:7892-7927.

2. Slack, J.M.W. Origin of stem cells in organogenesis. Science (New York, N.Y.). 2008; 322:1498-1501.

3. Jan Barfoot, Donald Bruce, Graeme Laurie, Nina Bauer, Janet Paterson and Mary Bownes. Text book of Stem cells science and ethics 1st edition, chapter one. 2016.

4. Dominici, M.et al. Minimal criteria for defining multipotent mesenchymal stromal cells. The International Society for Cellular Therapy position statement. Cytotherapy. 2006; 8:315-317.

5. Egusa, H. et al. Stem cells in dentistry - Part I: Stem cell sources. Journal of Prosthodontic Research. 2012; 56:151-165.

6. Liu, W. et al. Canonical Wnt signaling differently modulates osteogenic differentiation of mesenchymal stem cells derived from bone marrow and from periodontal ligament under inflammatory conditions. Biochimica et biophysica acta. 2014; 1840:1125-34.
7. Kaku, M. et al. Identification and characterization of neural crest-derived cells in adult periodontal ligament of mice. Archives of Oral Biology. 2012; 57:1668-1675.

8. Iwasaki, K. et al. Periodontal regeneration using periodontal ligament stem cell transferred amnion. Tissue engineering. 2014; Part A, 20:693-704.

9. Zhu, W. et al. Comparison of the properties of human CD146+ and CD146- periodontal ligament cells in response to stimulation with tumour necrosis factor. Archives of Oral Biology. 2013; 58:1791-1803.

10. Pisciotta, A. et al. Human Dental pulp stem cells (hDPSCs): isolation, enrichment and comparative differentiation of two sub-populations. BMC Developmental Biology. 2015;15(1), p.14.

11. Li, Z. CD133: a stem cell biomarker and beyond. Experimental hematology \& oncology. 2013; 2(1), p.17.

12. Gordon JAR, Tye CE, Sampaio AV, Underhill TM, Hunter GK, Goldberg HA. Bone sialoprotein expression enhances osteoblast differentiation and matrix mineralization in vitro. Bone. 2007; 41:462-473.

13. Xu L, Anderson AL, Lu QH, Wang JX. Role of fibrillar structure of collagenous carrier in bone sialoproteinmediated matrix mineralization and osteoblast differentiation. Biomaterials. 2007; 28:750-761.

14. Noda M, Yoon K, Rodan GA, Koppel DE. High Lateral Mobility of Endogenous and Transfected Alkaline-Phosphatase - a Phosphatidylinositol-Anchored MembraneProtein. Journal of Cell Biology. 1987; 105:1671-1677.

15. Low MG. Glycosyl-Phosphatidylinositol - a Versatile Anchor for Cell-Surface Proteins. Faseb Journal.1989; 3:1600-1608.

16. Abe T, Hara Y, Abe Y, Aida Y, Maeda K. Serum or growth factor deprivation induces the expression of alkaline phosphatase in human gingival fibroblasts. Journal of Dental Research. 1998; 77:1700-1707.

17. Harris H. The Human Alkaline-Phosphatases - What We Know and What We Dont Know. Clinica ChimicaActa. 1990; 186:133-150.

18. Leung KS, Fung KP, Sher AHL, Li CK, Lee KM. Plasma BoneSpecific Alkaline-Phosphatase as an Indicator of Osteoblastic Activity. Journal of Bone and Joint SurgeryBritish Volume.1993; 75:288-292.

19. Piattelli A, Scarano A, Corigliano M, Piattelli M. Effects of alkaline phosphatase on bone healing around plasmasprayed titanium implants: A pilot study in rabbits. Biomaterials.1996; 17:1443-1449. 
20. Karina Gottardello Zecchin, Rogério da Silva Jorge and Jacks Jorge. A new method for extraction of mandibular first molars in rats. Braz J Oral Sci. 2007; Vol. 6 - Number 21.

21. Jakub Suchánek, Klára Zoe Browne, Tereza Suchánková Kleplová and Yvona Mazurová. Protocols for DentalRelated Stem Cells Isolation, Amplifi cation and Differentiation. B. Zavan, E. Bressan (eds.), Dental Stem Cells: Regenerative Potential, Stem Cell Biology and Regenerative Medicine. 2016.

22. Ormerod MG. Analysis of DMA-general methods. Flow cytometry: a practical approach. Oxford, Oxford University Press, 2000; May (229): 83.

23. Zheng W., Wang, S., Ma, D., Tang, L., Duan, Y. and Jin, Y. Loss of proliferation and differentia-tion capacity of aged human periodontal ligament stem cells and rejuvenation by exposure to theyoung extrinsic environment. Tissue Engineering. 2009; Part A 15:2363-2371.

24. Park J-C, Kim J-M, Jung I-H, Kim JC, Choi S-H, Cho K-S, and Kim C-S. Isolation and characterization of human periodontal ligament (PDL) stem cells (PDLSCs) from the inflamed PDL tissue: in vitro and in vivo evaluations. J Clin Periodontol. 2011; 38:721-731.

25. Shabbir A, Cox A, Rodriguez-Menocal L, Salgado M. and Badiavas E Van. Mesenchymal Stem Cell Exosomes Induce Proliferation and Migration of Normal and Chronic Wound Fibroblasts, and Enhance Angiogenesis In Vitro. Stem Cells Dev. 2015; 24(14):1635-1647.

26. Hasegawa N, Kawaguchi H, Hirachi A, Takeda K, Mizuno N. and Nishimura M. Behavior of transplanted bone marrow-derived mesenchymal stem cells in periodontal defects. J Periodontol. 2006; 77(6):1003-7.

27. Haase HR, Clarkson RW, Waters MJ. And Bartold PM. Growth factor modulation of mitogenic responses and proteoglycan synthesis by human periodontal fibroblasts. J Cell Physiol. 1998; 174:35-361.

28. Tatullo, M., Marrelli, M. \& Paduano, F. The regenerative medicine in oral and maxillofacial surgery: the most important innovations in the clinical application of mesenchymal stem cells. International journal of medical sciences. 2015;12:72-77.

29. Navabazam AR, Sadeghian Nodoshan F, Sheikhha MH, Miresmaeili SM, Soleimani M, Fesahat F. Characterization of mesenchymal stem cells from human dental pulp, preapical follicle and periodontal ligament. Iran J Reprod Med. 2013; 11(3):235-42.

30. Silverio KG, Rodrigues TL, Coletta RD, Benevides L, Da Silva JS, Casati MZ. Mesenchymal stem cell properties of periodontal ligament cells from deciduous and permanent teeth. J Periodontol. 2010; 81(8):1207-15.

31. Wang L, Shen H, Zheng W, Tang L, Yang Z, Gao Y, et al. Characterization of stem cells from alveolar periodontal ligament. Tissue Eng Part A. 2011; 17(7-8):1015-26.

32. Alge DL, Zhou D, Adams LL, Wyss BK, Shadday MD, et al. Donor matched comparison of dental pulp stem cells and bone marrow-derived mesenchymal stem cells in a rat model. J Tissue Eng Regen Med. 2010; 4: 73-81.

33. Eleuterio, E. et al. Proteome of human stem cells from periodontal ligament and dental pulp. PLoS One. 2013; 8:71-101.

34. Chung CH, Golub EE, Forbes E, Tokuoka T, Shapiro IM. Mechanism of action of beta-glycerophosphate on bone cell mineralization. Calcif Tissue Int. 1992; 51:305-11.

35. Piattelli A, Scarano A, Corigliano M, Piattelli M. Effects of alkaline phosphatase on bone healing around plasmasprayed titanium implants: A pilot study in rabbits. Biomaterials. 1996; 17:1443-1449.

36. Ohazama A, Modina SA, Miletich I, Sharpe PT. Stemcell based tissue engineering of marine teeth. J Dent Res. 2004; 83:518-522.

37. Kim RH, Shapiro HS, Li JJ, Wrana JL, Sodek J. Characterization of the human bone sialoProtein (BSP) Gene and its Promoter Sequence. Matrix Biology. 1994; 14:31-40.

38. Boskey AL. Matrix proteins and mineralization: an overview. Connect Tissue Res. 1996; 35:357-363. 